\title{
Finding the Needle in the Haystack-An Unusual Case of Astasia-Abasia Following Sacro-Iliac Joint Injection for Chronic Low Back Pain
}

\author{
Gurmukh Das Punshi ${ }^{(0)}$, Andrew Purcell ${ }^{(0}$, Camillus Power* ${ }^{*}$ \\ Department of Anaesthesiology, Critical Care and Pain Medicine, Tallaght University Hospital, Dublin, Ireland \\ Email: *camillus.power@tuh.ie
}

How to cite this paper: Punshi, G.D., Purcell, A. and Power, C. (2021) Finding the Needle in the Haystack-An Unusual Case of Astasia-Abasia Following Sacro-Iliac Joint Injection for Chronic Low Back Pain. Pain Studies and Treatment, 9, $1-6$.

https://doi.org/10.4236/pst.2021.91001

Received: December 3, 2020

Accepted: January 23, 2021

Published: January 26, 2021

Copyright $\odot 2021$ by author(s) and Scientific Research Publishing Inc. This work is licensed under the Creative Commons Attribution International License (CC BY 4.0).

http://creativecommons.org/licenses/by/4.0/

\begin{abstract}
We present the case of a 60 yrs old male who, following a routine, uneventful, fluoroscopically guided L5/S1 facet joint rhizolysis and sacro-iliac joint (SIJ) injection developed an unexpected inability to stand or walk, a condition known as astasia-abasia. Initial concern had been that this neurological phenomenon was as a result of complications of his chronic pain intervention. Despite an essentially normal neurological examination and dedicated battery of neurological imaging and special testing, no cause was identified. Over a 7-day period of in-patient admission and physical rehabilitation symptoms resolved entirely. In the course of the workup for this episode, it was suggested that the phenomenon was the result of a side effect of dexamfetamine, an agent that had been prescribed for the patient by his neurologist for treatment of his narcolepsy. On the back of this episode, this treatment was discontinued by his neurologist following an outpatient consultation. Subsequent repeated SIJ injections were entirely uneventful and the patient experienced no further occurrences of this phenomenon.
\end{abstract}

\section{Keywords}

Chronic Low Back Pain, Sacroiliac Joint Injection, Dexamfetamine, Astasia-Abasia

\section{Introduction}

Chronic low back pain represents the second leading cause of disability worldwide with major social welfare and economic impact. Facet joint and SIJ me- 
diated pain are thought to account for $15 \%$ and $10 \%$ of cases respectively [1].

Classically, SIJ pain is often described as pain occurring below the level of L5. When indicated, an SIJ injection can be used to aid in the diagnosis and treatment of SIJ pain. This procedure involves introducing a local anaesthetic or a mixture of local anaesthetic and corticosteroid into the SIJ. Innervation of the SIJ can involve the dorsal rami of L5 and S1 [2]. For this reason, it is common practice to concomitantly block the median branch nerve of the dorsal ramus at this level owing to its potential contribution to SIJ mediated pain. Once sustained and adequate pain relief is achieved, the patient can return to normal function or a physical therapy regimen can be implemented [3]. Therapeutic SI joint injection can provide prolonged pain relief for patients suffering from SI joint dysfunction [4]. Significant improvement of up to 9 months' duration can be seen in over $60 \%$ of patients treated with SIJ steroid injection [3]. It is classically a well-tolerated procedure. Localised, transient post-procedural tenderness is common but more serious complications such as trauma to the nerves, haematoma, sciatic palsy, abscess, and systematic infection are, thankfully, more rare [4].

We present a case involving a neurological phenomenon occurring in the acute, post-procedural setting. Our patient, following routine, uneventful SIJ and facet joint injections, developed an unexpected inability to stand or walk, despite demonstrable sparing of motor function, a condition known as astasia-abasia. Historically astasia-abasia was thought to be a conversion disorder however the literature described the possibility of it being a neurological manifestation of both psychogenic and non-psychogenic causes [5]. Non-psychogenic astasia-abasia has been associated with CNS lesions affecting the pontomesencephalic region, thalamus, corpus callosum, or cingulate cortex. It has also been described in one case report as being attributed to alcohol consumption [6].

\section{Case Details}

Our patient was a 60-year-old male with a 10-year history of axial lower back pain with treatment limited to conservative management in the form of physiotherapy programmes and pharmacological therapies (currently taking gabapentin $600 \mathrm{mg}$ three times a day; previously failed trials of NSAIDs and tramadol). His past medical history was significant for a seven-year history of obstructive sleep apnoea (OSA), for which he was being managed with nocturnal continuous positive airway pressure (CPAP). Of note, the patient also suffered from narcolepsy, for which his neurologist had prescribed oral dexamfetamine $10 \mathrm{mg}$ twice daily. Additionally, having had a previous history of unexplained collapse/seizures he was also prescribed levetiracetam 500 mg twice daily. He had no known drug allergies.

As part of the work up for his chronic low back pain he had undergone an MRI of his lumbosacral spine that had shown mild facet joint arthropathy with a broad based disc bulge with no stenosis at the level of L3/4. At the level of L4/5, 
there was evidence of a moderate facet joint hypertrophy and a small central disc bulge with no evidence of vertebral canal stenosis. At the level of L5/S1 there was evidence of a small central osteophyte bulge without nerve root compression.

Following out-patient clinic consultation, where a thorough history and physical examination was conducted, the patient was scheduled for and underwent an elective, fluoroscopically guided, right sided L4/5, L5/S1 facet joint and SIJ injection with $0.5 \%$ levobupivacaine and $40 \mathrm{mg}$ total of depo-medrone (methylprednisolone). The procedure was completed under local anaesthesia and was entirely uneventful. Prior to being discharged home the patient was reviewed on the day ward, as was routine for all day case patients. At this time the patient informed the team that he had an inability to stand or walk. A decision was made to admit the patient for observation and further assessment/management.

A full neurological examination was conducted that revealed no apparent deficits; apart from the subjective inability to stand or walk, the motor and sensory components of the lower limb examination were entirely normal. Routine blood work (Full blood count, renal and bone profiles, liver function tests and inflammatory markers) was unremarkable, with all test results being within the normal range of limits. A formal neurology consult was arranged, the findings of which corroborated our own clinical findings. A battery of neurological imaging and special testing was undertaken. These included a CT and subsequent MRI brain scan, both of which were unremarkable, an MRI lumbosacral spine that demonstrated no interval changes compared to prior imaging findings mentioned previously and somatosensory evoked potentials that did demonstrate an approximate deficit of $50 \%$ on the right lower limb compared to the left lower limb, however the aetiology of this deficit was however unclear owing to the fact that there was previously documented degenerative lumbosacral spine changes that could have accounted for this.

In light of these findings a neuropsychology assessment was sought to investigate whether a stress related phenomena might be contributing to his symptomatology. The neuropsychology service concluded that there was no evidence of a stress disorder. He also underwent in-patient physical rehabilitation with our physiotherapy colleagues. Within seven days the patient had recovered enough function with their input that he was deemed suitable for discharge with outpatient follow up arranged thereafter.

\section{Discussion}

Lower Back pain (LBP) is one of the most common symptoms and conditions for which medical consultation is sought [7]. One source of chronic LBP is lumbar facet joint degeneration. It has an estimated incidence of $15 \%-45 \%$ among patients with LBP [8]. The SIJ is the suspected culprit in an estimated $10 \%-15 \%$ of cases of chronic LBP [9]. Interventional techniques for these causes of pain have been shown to have a positive effect on pain relief and improved quality of life in chronic LBP patients [10].

In our case the patient developed an unexpected complication whereby he ex- 
perienced a subjective inability to stand or walk (a condition known as astasia-abasia) following a seemingly uneventful procedure. Fluoroscopically directed facet joint and SIJ injections are generally regarded as being well tolerated and low risk procedures associated with an extremely low incidence of major complications, although minor side effects such as localised tenderness, small vessel puncture and minor bruising are relatively more common [11].

Immediate concern would have been regarding the possibility of an iatrogenic epidural haematoma. This is a rare but potentially anticipated complication of this procedure having been previously described in case reports involving facet joint injections [8]. Another possible differential following SIJ injection, transient sciatic nervy palsy, has also been described in the literature [12]. In this instance however both of these complications would be less likely to be the culprit owing to an essentially normal objective neurological examination. It is also unlikely that iatrogenic sciatic nerve injury occurred in our case as the patient never complained of any pain during or after procedure which would be expected in this circumstance. Nerve ischaemia secondary to inadvertent injection into inferior gluteal artery was also a possibility given it is an adjacent structure, but it is also unlikely due to recovery of symptoms within a short period of time (one week). Prolonged effects of local anaesthetic administered during the procedure was also possible given that perineural pooling in proximity to sciatic nerve following sacroiliac joint injection is theoretically possible. This again was deemed to be unlikely owing to the lack of any objective motor or sensory deficit on neurological examination of the affected lower limb.

The absence of any clear iatrogenic cause of this episode had these authors seeking out more esoteric explanations for this presentation. Casting the net wider for an explanation, we investigated whether or not some pre-existing medication may have accounted for these symptoms. Indeed, it would appear that dexamfetamine can produce seizure-like episodes (of which the patient had a documented history of and was prescribed levetiracetam) while also possibly producing weakness, coordination and gait disturbances as described by the patient [13] [14]. With this, it is not unreasonable to conclude that in the absence of any neurological cause (physical or psychological), the timing of the presentation of astasia-abasia in line with administration of these chronic pain interventions was coincidental and more likely to potentially be a manifestation of a possibly rare side effect of dexamfetamine. A review of the literature was unable to identify any previous cases of astasia-abasia attributed to this drug.

Upon review in our outpatient clinic (eight weeks after his discharge) it was evident that there had been no recurrence of these neurological symptoms and that his back pain was thankfully better to the extent that his sleep, mood and physical functionality had all subjectively improved. With this in mind, he was referred back to his neurologist, the source of his dexamfetamine prescription. Here, the dexamfetamine was discontinued in light of how stressful this episode had been for the patient. Interestingly, the patient subsequently underwent two further interval SIJ injection procedures while no longer taking the suspected 
offending medication and experienced no further unusual episodes. Overall, he described being satisfied with this outcome.

\section{Conclusions}

Facet joint and sacroiliac joint injections are generally safe procedures without major complications; nevertheless, clinicians should be aware of the potential risk of serious complications. It is important to acknowledge that these complications can occur rarely.

While practitioners need to be vigilant about possible mechanisms for nerve damage when undertaking these procedures, this case report highlights that sometimes we cannot definitively explain why a clinical event has happened and outside the box thinking is required to establish a satisfactory differential diagnosis.

Regardless of cause, patients should be assessed, investigated and supported throughout their recovery.

\section{Ethical Statement}

Full, informed patient consent to submit this case for academic publication was provided by the patient.

\section{Conflicts of Interest}

The authors declare no conflicts of interest regarding the publication of this paper.

\section{References}

[1] Barnsley, L., Lord, S.M., Wallis, B.J. and Bogduk, N. (1995) The Prevalence of Chronic Cervical Zygapophysial Joint Pain after Whiplash. Spine, 20, 20-25.

[2] Forst, S.L., Wheeler, M.T., Fortin, J.D. and Vilensky, J.A. (2006) The Sacroiliac Joint: Anatomy, Physiology and Clinical Significance. Pain Physician, 9, 61-67.

[3] Kisnher, S., Faciane, J.L., et al. (2019) Sacroiliac Joint Injection. Medscape. https://emedicine.medscape.com/article/103399-overview

[4] Wu, L., Tafti, D. and Varacallo, M. (2020) Sacroiliac Joint Injection. StatPearls. https://www.ncbi.nlm.nih.gov/books/NBK513245/

[5] Vercueil, L. (2010 ) Astasie-Abasie: Causes psychogènes et non psychogènes (AstasiaAbasia: Psychogenic and Non-Psychogenic Causes). Revue Neurologique, 166, 221-228.

[6] Han-Joon, K., Jee-Young, L. and Beom, S.J. (2010) Episodic Astasia-Abasia Associated with Hyperperfusion in the Subthalamic Region and Dorsal Brainstem. Neurology Asia, 15, 279-281.

[7] Allegri, M., Montella, S., Salici, F., et al. (2016) Mechanisms of Low Back Pain: A Guide for Diagnosis and Therapy. F1000 Research, 5, 1530.

[8] Velickovic, M. and Ballhause, T.M. (2016) Delayed Onset of a Spinal Epidural Hematoma after Facet Joint Injection. SAGE Open Medical Case Reports, 4, 1-5.

[9] Kishner, S. and Schraga, E.D. (2017) Sacroiliac Joint Injection Technique. https://emedicine.medscape.com/article/103399-technique- 
[10] Lord, S.M., Barnsley, L., Wallis, B.J., McDonald, G.J. and Bogduk, N. (1996) Percutaneous Radio-Frequency Neurotomy for Chronic Cervical Zygapophyseal-Joint Pain. The New England Journal of Medicine, 335, 1721-1726.

[11] Manchikanti, L., Malla, Y., Wargo, B.W., Cash, K.A., Pampati, V. and Fellows, B. (2012) Complications of Fluoroscopically Directed Facet Joint Nerve Blocks: A Prospective Evaluation of 7,500 Episodes with 43,000 Nerve Blocks. Pain Physician, 15, E143-E150.

[12] Visser, L.H., Nijssen, P.G., Tijssen, C.C., Van Middendorp, J.J. and Schieving, J. (2013) Sciatica-Like Symptoms and the Sacroiliac Joint: Clinical Features and Differential Diagnosis. European Spine Journal, 22, 1657-1664.

[13] Electronic Medicines Compendium (EMC) (2020) Dexamfetamine Sulfate $5 \mathrm{mg}$ Tablets Summary of Products Characteristics (SmPC). https://www.medicines.org.uk/emc/product/11004/smpc

[14] Electronic Medicines Compendium (EMC) (2020) Dexamfetamine Sulfate $5 \mathrm{mg}$ Tablets Patient Information Leaflet (PIL).

https://www.medicines.org.uk/emc/product/11004/pil 